\title{
Nephrotic Range Proteinuria in Renal Transplantation: Clinical and Histologic Correlates in a 10-year Retrospective Study
}

\author{
R. Leal ${ }^{a, \star}$, H. Pinto ${ }^{a}$, A. Galvão ${ }^{a}$, L. Santos ${ }^{a}$, C. Romãozinho ${ }^{a}$, F. Macário $^{a}$, R. Alves ${ }^{a}$, J. Pratas ${ }^{a}$, \\ V. Sousa ${ }^{b}$, C. Marinho ${ }^{b}$, L. Prado e Castro ${ }^{b}$, M. Campos $^{a}$, A. Mota ${ }^{c}$, and A. Figueiredo ${ }^{c}$ \\ ${ }^{a}$ Nephrology Department, Centro Hospitalar e Universitário de Coimbra, Coimbra, Portugal; ${ }^{\circ}$ Anatomic Pathology Department, Centro \\ Hospitalar e Universitário de Coimbra, Coimbra, Portugal; and 'Urology and Renal Transplantation Department, Centro Hospitalar e \\ Universitário de Coimbra, Coimbra, Portugal
}

\begin{abstract}
Introduction. There is a high incidence of nephrotic proteinuria in renal transplant recipients, which is an accurate predictor of graft loss. Despite this, its histologic correlates and prognostic implications are still not well characterized. We assessed the clinical and histological correlates of kidney transplantation patients with nephrotic range proteinuria.

Methods. We have retrospectively analyzed clinical and histological data from 50 kidney transplantation biopsy specimens from 44 renal transplant recipients with nephrotic range proteinuria between 2006 and 2015. The median follow-up time was 93 months (range, 14 months to 190 months).

Results. The mean age of the patients was $45.2 \pm 13.7$ years and our cohort included $86 \%$ recipients of deceased-donor grafts. The maintenance immunosuppressive regimen included calcineurin inhibitors in $68 \%$ and mammalian target of rapamycin inhibitors in $32 \%$ of patients. The average proteinuria was $6.9 \pm 3.8 \mathrm{~g} / \mathrm{d}$ and $52 \%$ of patients presented with nephrotic syndrome. The main histological findings were transplant glomerulopathy $(22 \%)$, de novo glomerular disease $(22 \%)$, and recurrence of primary disease $(22 \%)$. Tubular atrophy and interstitial fibrosis was present in $78 \%$ of the biopsy specimens. Thirty-one patients $(62 \%)$ lost the graft at follow-up. There was no statistically significant difference between the histologic diagnosis nor the proteinuria levels and the outcome of the graft.

Conclusions. The main causes of nephrotic range proteinuria in patients undergoing biopsy were transplant glomerulopathy, recurrence of the underlying disease, and de novo glomerulonephritis. Nephrotic range proteinuria was related to a high rate of graft loss.
\end{abstract}

$\mathbf{N}$ EPHROTIC proteinuria in kidney transplant (KT) patients is one of the most accurate predictors of graft loss and patients with nephrotic syndrome have a 5-year graft survival rate significantly lower than patients without proteinuria [1,2]. The 2009 Kidney Disease: Improving Global Outcomes (KDIGO) clinical practice guidelines on KT suggest frequent measuring of urine protein excretion and renal allograft biopsy for new-onset proteinuria or unexplained nephrotic-range proteinuria [3]. Unfortunately, histologic correlates and prognostic implications of post-transplantation proteinuria are still not well characterized and evidence-based therapies are limited [4].
Our goal was to assess the clinical and histological correlates of KT patients with nephrotic range proteinuria in our center.

\section{PATIENTS AND METHODS}

An observational retrospective single-center study was performed from January 1, 2006, to December 31, 2016. Among the $450 \mathrm{KT}$ biopsies performed during that period in our center, we selected all

*Address correspondence to Rita Leal, MD, Nephrology Department, Centro Hospitalar e Universitário de Coimbra, Praceta Prof. Mota Pinto, 3000-075 Coimbra, Portugal. E-mail: rita.gcleal@gmail.com

(c) 2017 Elsevier Inc. All rights reserved. 230 Park Avenue, New York, NY 10169 
the biopsy specimens of patients who presented 24-hour proteinuria $\geq 3.5 \mathrm{~g}$ at the time of the biopsy. A total of 50 biopsy specimens from 44 recipients were selected. The 2011 Banff classification for renal transplant biopsy was used for diagnostic categorization.

Patient records were reviewed for clinical features, response to treatment, and graft outcome. The median follow-up time was 93 months (range, 14 months to 190 months).

Remission was defined as protein excretion of less than $0.5 \mathrm{~g} / \mathrm{d}$ for 2 consecutive assays.

Data was analyzed using SPSS statistics software (IBM SPSS Statistics for Windows, version 21, IBM Corp., Armonk, NY, USA). Comparisons used the $\chi^{2}$ test for categorical variables and Student $t$ test or the Mann-Whitney U test for continuous variables, as appropriate. Results were considered statistically significant at $P<.05$.

\section{RESULTS}

\section{Clinical Characteristics}

The 44 recipients evaluated included $64 \%(\mathrm{~N}=32)$ males with a mean age of $45.2 \pm 13.7$ years. It was the first transplant for $84 \%$ of the patients $(\mathrm{N}=42)$, and $86 \%$ $(\mathrm{N}=43)$ received a cadaveric graft, of which $46 \%$ fulfilled marginal donor criteria. Regarding immunosuppression, induction with basiliximab was performed in $54 \%$ of the recipients $(\mathrm{N}=27)$. The maintenance immunosuppressive included calcineurin inhibitors in $68 \%$ and mammalian target of rapamycin (mTOR) inhibitors in $32 \%$ of patients (Table 1).

\begin{tabular}{|c|c|}
\hline \multicolumn{2}{|l|}{ Demographic Features } \\
\hline Age (y) (mean $\pm \mathrm{SD}$ ) & $45.2 \pm 13.7$ \\
\hline Male gender & $\mathrm{N}=32,64 \%$ \\
\hline Cadaveric donor & $\mathrm{N}=43,86 \%$ \\
\hline Expanded criteria donor & $\mathrm{N}=23,46 \%$ \\
\hline $\mathrm{BMI}\left(\mathrm{kg} / \mathrm{m}^{2}\right)($ mean $\pm \mathrm{SD})$ & $25.3 \pm 3.8$ \\
\hline Diabetes mellitus & $\mathrm{N}=8,16 \%$ \\
\hline Hypertension & $\mathrm{N}=30,60 \%$ \\
\hline \multicolumn{2}{|l|}{ Primary Kidney Disease } \\
\hline Undetermined & $\mathrm{N}=15,30 \%$ \\
\hline Chronic glomerulonephritis (unspecified) & $\mathrm{N}=14,28 \%$ \\
\hline Diabetic nephropathy & $\mathrm{N}=4,8 \%$ \\
\hline IgA nephropathy & $\mathrm{N}=4,8 \%$ \\
\hline Nephroangiosclerosis & $\mathrm{N}=3,6 \%$ \\
\hline Focal segmental glomerulosclerosis & $\mathrm{N}=3,6 \%$ \\
\hline Polycystic Kidney Disease & $\mathrm{N}=3,6 \%$ \\
\hline Membranous nephropathy & $\mathrm{N}=2,4 \%$ \\
\hline Type 1 primary hyperoxaluria & $\mathrm{N}=2,4 \%$ \\
\hline HLA mismatches (mean \pm SD) & $3.0 \pm 1.2$ \\
\hline \multicolumn{2}{|l|}{ Maintenance Immunosuppression } \\
\hline Regimen including steroids & $\mathrm{N}=40,80 \%$ \\
\hline Calcineurin inhibitors & $\mathrm{N}=34,68 \%$ \\
\hline mTOR inhibitors & $\mathrm{N}=15,30 \%$ \\
\hline \multicolumn{2}{|l|}{ Post-transplantation Clinical Events } \\
\hline Acute rejection & $N=6,12 \%$ \\
\hline Chronic rejection & $\mathrm{N}=8,16 \%$ \\
\hline Previous infection with hospital admission & $\mathrm{N}=5,10 \%$ \\
\hline
\end{tabular}

Abbreviations: SD, standard deviation; BMI, body mass index; IgA immunoglobulin A; mTOR, mammalian target of rapamycin.
The mean basal creatinine value was $1.67 \pm 0.67 \mathrm{mg} / \mathrm{dL}$ and the mean creatinine value at the time of the biopsy was $2.53 \pm 1.14 \mathrm{mg} / \mathrm{dL}$. The mean 24-hour proteinuria was $6.9 \pm$ $3.8 \mathrm{~g}$ and 26 patients $(52 \%)$ presented with concomitant nephrotic syndrome. Regarding donor-specific HLA antibodies, they were positive in 15 recipients at the time of graft biopsy $(30 \%)$.

\section{Allograft Histology}

The mean time post-transplantation at biopsy was 44 months (range, 24 months to 102.3 months). Histological findings of the 50 biopsy specimens are displayed in Table 2. The main primary histologic diagnoses were transplant glomerulopathy, recurrent glomerular diseases, and de novo glomerular diseases. Of the 11 patients with transplant glomerulopathy, 7 presented with positive $\mathrm{C} 4 \mathrm{~d}$ staining and donor-specific HLA antibodies (7 of 11, 64\%) and were diagnosed with chronic humoral rejection. Regarding de novo glomerular diseases, 3 patients had undetermined cause for primary chronic kidney disease, whereas the others had chronic kidney disease due to nephroangiosclerosis $(\mathrm{N}=2)$, diabetic nephropathy $(\mathrm{N}=3)$, immunoglobulin $\mathrm{A}(\operatorname{Ig} \mathrm{A})$ nephropathy $(\mathrm{N}=2)$, and membranous nephropathy $(\mathrm{N}=1)$, with a different histological diagnosis on the transplant biopsy.

Interstitial fibrosis and tubular atrophy with glomerular sclerosis was present in 39 biopsy specimens (78\%) but only in 8 specimens represented the primary diagnosis in the context of chronic allograft nephropathy.

\section{Treatment}

Maintenance therapy with angiotensin-converting enzyme inhibitors or angiotensin receptor blockers was performed in 16 patients (32\%) with chronic allograft nephropathy,

Table 2. Findings in 50 Transplantation Biopsy Specimens in 44 Patients With Post-transplantation Nephrotic Proteinuria

\begin{tabular}{lrr}
\hline \multicolumn{1}{c}{ Primary Diagnoses } & N & $\%$ \\
\hline Recurrent glomerular diseases & 11 & 22 \\
Diabetic nephropathy & 3 & \\
IgA nephropathy & 3 & \\
FSGS & 3 & \\
Primary hyperoxaluria & 2 & \\
Transplant glomerulopathy & 11 & 22 \\
De novo glomerular disease & 11 & 22 \\
Membranoproliferative GN with full house IF & 4 & \\
FSGS & 3 & \\
Membranous GN & 1 & \\
Mesangioproliferative GN & 1 & \\
Collapsing FSGS & 1 & \\
Crescentic GN & 1 & \\
FSGS + IFTA & 8 & 16 \\
FSGS + acute rejection & 5 & 10 \\
Other & 4 & 8 \\
Total & 50 & 100 \\
\hline
\end{tabular}

Abbreviations: IgA, immunoglobulin A; FSGS, focal segmental glomerulosclerosis; GN, glomerulonephritis; IF, immunofluorescence; IFTA, interstitial fibrosis and tubular atrophy. 
and transplant glomerulopathy with important interstitial fibrosis and tubular atrophy. Increased immunosuppression was the approach in 18 patients $(36 \%)$ with transplant glomerulopathy, recurrent glomerular diseases, and de novo glomerular diseases. Methylprednisolone pulses were performed in 9 patients $(18 \%)$ including acute rejection imposed in chronic allograft nephropathy and de novo glomerular diseases. Therapeutic plasma exchange was performed in 5 patients (10\%): 2 patients with antibody mediated rejection and 3 patients with recurrent focal and segmental sclerosis. Two patients received rituximab including 1 patient with membranoproliferative glomerulonephritis and 1 patient for chronic antibody mediated rejection.

\section{Outcome}

The median follow-up time was 93 months (range, 14 months to 190 months). Complete remission of the nephrotic proteinuria was observed in 5 patients $(11 \%)$, three of them with de novo glomerular disease and two with recurrent glomerular disease. Thirty patients $(68 \%)$ are dialysis-dependent, and there were three deaths registered. There was no difference between the immunosuppressive regimen used and the levels of proteinuria. There was no statistically significant difference between the histologic diagnosis nor the proteinuria levels and the outcome of the graft.

\section{DISCUSSION}

Previous studies report an average prevalence of 24-hour proteinuria $>3 \mathrm{~g} / 24$-hour in KT recipients of $15 \%$ [5-7]. The present study reports post-transplantation proteinuria in $22.5 \%$ patients submitted to graft biopsies in a 10 -year period. This high incidence might be related to the study design, as the general population includes patients undergoing biopsy, conditioning a selection bias.

Regarding etiology, the main histological findings in our report are concordant with the existing literature: recurrent glomerular diseases, de novo glomerulopathy, and transplant glomerulopathy [4,7].

In the KT population, the evidence base for the management of proteinuria is lacking for most cases, but routine monitoring of proteinuria as part of follow-up is recommended. Renin-angiotensin-aldosterone system blockade results in a significant reduction in proteinuria, but this benefit is frequently accompanied by a significant reduction in glomerular filtration rate and there is no evidence of improved patient or graft survival [4]. Some studies have noted an association between proteinuria and the use of sirolimus, especially in patients switched from calcineurin inhibitors [8]. We did not find any significant relation between the use of mTOR inhibitors and the level of proteinuria.

Our study reported a high rate of graft loss at time of follow up $(69 \%)$. This might be related to the high prevalence of interstitial fibrosis and tubular atrophy found in our biopsy specimens, indicating chronic lesions regardless the glomerular changes. Also, the time of follow up was very variable and the majority of the patients were transplanted for more than 5 years at the time of biopsy. Although proteinuria is strongly associated with graft survival, other factors, such as donor age, may be equally significant $[4,5,9]$.

\section{CONCLUSION}

In our report, the main causes of nephrotic range proteinuria in patients undergoing biopsy were transplant glomerulopathy, recurrence of the underlying disease, and de novo glomerulonephritis. Nephrotic range proteinuria was related to a high rate of graft loss.

\section{REFERENCES}

[1] Barnas U, Schmidt A, Haas M, et al. Parameters associated with chronic renal transplant failure. Nephrol Dial Transplant 1997;12:82-5.

[2] Reichel H, Zeier M, Ritz E. Proteinuria after renal transplantation: pathogenesis and management. Nephrol Dial Transplant 2004;12:301-4.

[3] The National Kidney Foundation's Kidney Disease Outcomes Quality Initiative (KDOQI). KDIGO clinical practice guideline for the care of kidney transplant recipients. Am J Transplant 2009;9:1-157.

[4] Shamseddin M, Knoll G. Posttransplantation proteinuria: an approach to diagnosis and management. Clin J Am Soc Nephrol 2011;6:1786-93.

[5] Fernandez-Fresnedo G, Escallada R, Rodrigo E, De Francisco A, Cotorruelo J, Sanz De Castro S, et al. The risk of cardiovascular disease associated with proteinuria in renal transplant patients. Transplantation 2002;73:1345-8.

[6] First M, Vaidya P, Maryniak R, Weiss M, Munda R, Fidler J, et al. Proteinuria following transplantation: correlation with histopathology and outcome. Transplantation 1984;38:607-12.

[7] Yakupoglu U, Baranowska-Daca E, Rosen D, Barrios R, Suki W, Truong L. Post-transplant nephrotic syndrome: a comprehensive clinicopathologic study. Kidney Int 2004;65: 2360-70.

[8] Huber S, Bruns C, Schimd G, et al. Inhibition of the mammalian target of rapamycin impedes lymphangiogenesis. Kidney Int 2007;71:771-7.

[9] Amer H, Fidler M, Myslak M, et al. Proteinuria after kidney transplantation, relationship to allograft histology and survival. Am J Transplant 2007;7(12):2748-56. 